\title{
Computational details
}

The absorption spectra have been calculated by the Time Depedent PBE0/6-311+G(2d,2p) calculations performed on geometries optimized at the PBE0 6-31+G(d,p) level. Numerical excited state geometry optimizations have been performed at the the TD/PBE0 6-31G(d) level, without any planarity constraint. All the gradients are smaller than $0.02 \mathrm{eV} / \mathrm{bohr}$ for the excited state geometry optimizations performed in vacuo and smaller than $0.05 \mathrm{eV} / \mathrm{bohr}$ for the geometry optimizations performed in aqueous solution and Hydrogen atom degrees of freedom are frozen at their value optimized in the gas phase.

In the calculation of the emission spectra, the coordination geometry of the four water molecules to the uracil molecule has been optimized freezing the uracil degrees of freedom at their values optimized in the aqueous solution.

Solvation model Bulk solvent effect on the UV spectra has been calculated by using the TD-PCM implementation described in ref. 18-19. However, it is well known that the proper description of solvent shifts in aqueous solutions solvents requires the explicit inclusion of water molecules belonging to the first solvation shell in the cluster, which is further embedded in the dielectric continuum mimicking bulk solvent. Taking into account experimental suggestions, ${ }^{15}$ we thus include four explicit water molecules. The most stable arrangement, shown in Figure 2, is in agreement with the indication of NMR experiments ${ }^{17}$ that no water molecule is strongly bonded to $\mathrm{C}_{5}$ and $\mathrm{C}_{6}$ carbon atoms and that $\mathrm{O}_{7}$ and $\mathrm{O}_{8}$ are coordinated by two and one water molecule, respectively. The results of a very recent Car-Parrinello dynamics ${ }^{18}$ suggest that the first coordination shell of uracil (up to $2.5 \AA$ ) is formed by 6 water molecules, 4 in the molecular plane (as in Figure 2) and two more or less perpendicular to it. Although a full description of the first solvation shell in solution requires, of course, dynamic considerations, a number of studies have confirmed that the PCM is able to model effectively the effect of water molecules that are more distant and/or not directionally bound to the carbonyl oxygen lone pairs. On the other hand, from a dynamic point of view, it could be useful to take into account all the water molecules of the cybotactic region. As a matter of fact, the evolution of uracil excited state strongly depends on the behavior of $\pi$ and $\pi^{*}$ orbitals, whose relative energy could be significantly influenced by the formation of short-lived adducts with perpendicularly bound solvent molecules.

All the calculations have been performed by using the Gaussian03 Package. (M. Frisch and al. Gaussian Inc. Pittsburgh PA) 

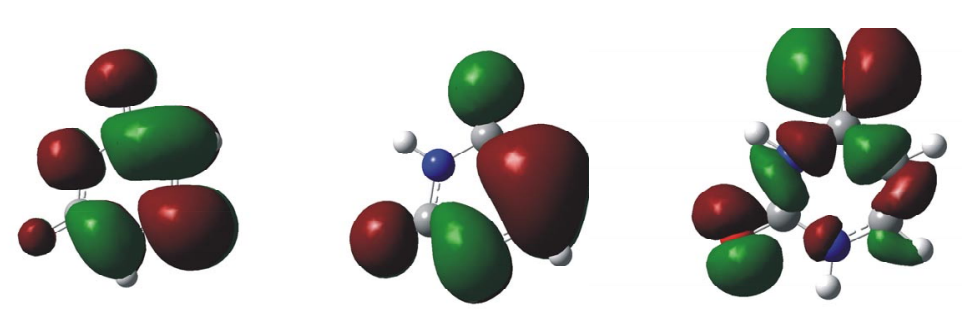

Figure S1: Schematic drawing of the frontier orbitals of uracil. LUMO (left), HOMO (center), HOMO-1 (right)

Table S1 Main geometrical parameters of the gas phase optimized geometries for the three lowest energy state of uracil. PBE0 and TDPBE0 computations.

\begin{tabular}{|c|c|c|c|c|c|}
\hline & \multicolumn{3}{|c|}{$\mathrm{S} 0$} & $\mathrm{~S} 1$ & $\mathrm{~S} 2$ \\
\hline & Exp & $6-31+\mathrm{G}(\mathrm{d}, \mathrm{p})$ & $6-31 \mathrm{G}(\mathrm{d})$ & $6-31 \mathrm{G}(\mathrm{d})$ & $6-31 \mathrm{G}(\mathrm{d})$ \\
\hline $\mathrm{N} 1-\mathrm{C} 2$ & 1.371 & 1.386 & 1.388 & 1.361 & 1.330 \\
\hline $\mathrm{C} 2-\mathrm{N} 3$ & 1.376 & 1.377 & 1.378 & 1.397 & 1.319 \\
\hline $\mathrm{N} 3-\mathrm{C} 4$ & 1.371 & 1.403 & 1.405 & 1.389 & 1.509 \\
\hline $\mathrm{C} 5-\mathrm{C} 6$ & 1.430 & 1.455 & 1.456 & 1.370 & 1.404 \\
\hline $\mathrm{C} 6-\mathrm{N} 1$ & 1.340 & 1.349 & 1.347 & 1.399 & 1.399 \\
\hline C2-07 & 1.358 & 1.370 & 1.369 & 1.398 & 1.431 \\
\hline C4-08 & $1.248 \$ \wedge \mathrm{a} \$$ & 1.218 & 1.215 & 1.319 & 1.295 \\
\hline N1-C2-N3 & 114.0 & 113.2 & 112.8 & 113.9 & 119.8 \\
\hline C2-N3-C4 & 126.7 & 128.1 & 128.5 & 123.8 & 125.7 \\
\hline N3-C4-C5 & 115.5 & 113.7 & 113.3 & 120.0 & 110.3 \\
\hline C4-C5-C6 & 118.9 & 119.6 & 119.8 & 118.7 & 124.8 \\
\hline C5-C6-N1 & 122.3 & 121.9 & 121.9 & 118.4 & 117.4 \\
\hline 07-C2-N1 & 123.7 & 122.7 & 122.7 & 125.0 & 120.1 \\
\hline 08-C4-N3 & 119.2 & 120.3 & 120.4 & 112.0 & 115.6 \\
\hline
\end{tabular}

Notes:a) double hydrogen bonded with the NH groups of a vicinal molecule in the crystal ( see R.F. Stewart, L.H. Jensen, Acta. Cryst. D 1967, 23, 1102) 
Table S2 Main geometrical parameters of the optimized geometries for the three lowest energy states of uracil in the aqueous solution. PBE0 and TD/PBE0 6-31G(d) computations.

\begin{tabular}{|l|l|l|l|}
\hline & S0 & S1 & S2 \\
\hline N1-C2 & 1.379 & 1.357 & 1.377 \\
\hline C2-N3 & 1.377 & 1.392 & 1.356 \\
\hline N3-C4 & 1.398 & 1.393 & 1.432 \\
\hline C4-C5 & 1.448 & 1.372 & 1.419 \\
\hline C5-C6 & 1.351 & 1.395 & 1.432 \\
\hline C6-N1 & 1.365 & 1.398 & 1.375 \\
\hline C2-07 & 1.220 & 1.223 & 1.238 \\
\hline C4-08 & 1.225 & 1.322 & 1.250 \\
\hline N1-C2-N3 & 113.4 & 114.6 & 117.0 \\
\hline C2-N3-C4 & 128.0 & 123.0 & 124.9 \\
\hline N3-C4-C5 & 113.8 & 120.2 & 114.2 \\
\hline C4-C5-C6 & 119.5 & 118.6 & 122.9 \\
\hline C5-C6-N1 & 122.0 & 125.0 & 125.1 \\
\hline $07-\mathrm{C} 2-\mathrm{N} 1$ & 123.1 & 124.4 & 119.7 \\
\hline $08-\mathrm{C} 4-N 3$ & 120.1 & 112.1 & 118.2 \\
\hline $\mathrm{O}_{\mathrm{w} 1-\mathrm{H} 1}$ & 1.853 & 1.908 & 1.760 \\
\hline $\mathrm{H}_{\mathrm{w} 1-\mathrm{O} 7}$ & 2.042 & 2.011 & 2.872 \\
\hline $\mathrm{H}_{\mathrm{w} 2-\mathrm{O} 2}$ & 1.976 & 1.989 & 2.012 \\
\hline $\mathrm{O}_{\mathrm{w} 3-\mathrm{H} 3}$ & 1.798 & 1.854 & 1.785 \\
\hline $\mathrm{H}_{\mathrm{w} 4-\mathrm{O} 8}$ & 1.792 & 1.998 & 1.742 \\
\hline & & & \\
\hline
\end{tabular}


Table S3. Lowest energy (in $\mathrm{eV}$ ) $\mathrm{n} / \pi^{*}$ and $\pi / \pi^{*}$ transitions in the gas phase. TD-PBE0 6$311+\mathrm{G}(2 \mathrm{~d}, 2 \mathrm{p})$ computations on PBE0 6-31+G(d,p) optimized geometries

\begin{tabular}{ccccc} 
Energy & $\begin{array}{c}\text { Oscillator } \\
\text { strength }\end{array}$ & $\begin{array}{c}\text { Kohn-Sham } \\
\text { orbitals involved }\end{array}$ & CASPT2 $^{\text {a }}$ & Experimental \\
\hline \multicolumn{5}{c}{$\mathrm{n} \rightarrow \pi^{*}$} \\
5.77 & 0.0000 & HOMO-1 $\rightarrow$ LUMO & $4.54(0.0)$ & $4.68^{\mathrm{b}}$ \\
5.94 & 0.0001 & HOMO-3 $\rightarrow$ LUMO & $6.00(0.0)$ & \\
6.36 & 0.0004 & HOMO-1 $\rightarrow$ LUMO+2 & $6.37(0.0)$ & \\
6.83 & 0.0001 & HOMO-3 $\rightarrow$ LUMO+2 & $6.95(0.0)$ & \\
\hline 5.24 & 0.1397 & HOMO $\rightarrow$ LUMO & $5.00(0.19)$ & $5.08^{\mathrm{c}}$ \\
6.06 & 0.0352 & HOMO-2 $\rightarrow$ LUMO & $5.82(0.08)$ & $6.05^{\mathrm{c}, \mathrm{d}}$ \\
6.55 & 0.1267 & HOMO $\rightarrow$ LUMO+2 & $6.46(0.29)$ & $6.63^{\mathrm{c}}$
\end{tabular}

Notes: a) From ref.14 Oscillator strengths are given in parentheses. b) in 1-methyl uracil (see Eaton, W.A.; Lewis, T.P. J. Chem. Phys. 1970, 53, 2164. c) From ref. 6 d) shoulder 
Table S4 Lowest energy electronic transitions for uracil in aqueous solution, computed according different computational scheme. TD-PBE0 6-311+G(2d,2p) computations on PBE0 6-31+G(d,p) optimized geometries. Oscillatore strength and experimental intensities are given in parentheses.

\begin{tabular}{cccc|c}
$\begin{array}{c}\text { Gas Phase }+ \\
4 \mathrm{H}_{2} \mathrm{O}\end{array}$ & $\begin{array}{c}\text { Gas Phase }+ \\
\mathrm{PCM}^{\mathrm{c}}\end{array}$ & $\begin{array}{c}\mathrm{PCM}+ \\
\mathrm{PCM}^{\mathrm{d}}\end{array}$ & $\begin{array}{c}\mathrm{PCM}+ \\
4 \mathrm{H}_{2} \mathrm{O}\end{array}$ & Experimental \\
\hline $4.98(0.00)^{\mathrm{a}}$ & $5.14(0.00)$ & $5.09(0.00)$ & $5.23(0.00)$ & \\
$5.25(0.14)^{\mathrm{b}}$ & $5.19(0.19)$ & $5.17(0.19)$ & $5.08(0.20)$ & $4.77^{\mathrm{e}, \mathrm{f}}(8100)$ \\
$6.32(0.05)^{\mathrm{b}}$ & $6.29(0.12)$ & $6.25(0.09)$ & $6.23(0.17)$ & $6.14^{\mathrm{e}} 6.05^{\mathrm{f}}(8800)$ \\
$6.36(0.10)^{\mathrm{b}}$ & $6.39(0.13)$ & $6.38(0.16)$ & $6.32(0.12)$ &
\end{tabular}

Notes: $\mathrm{n} \rightarrow \pi^{*}$ transition, b) $\pi \rightarrow \pi^{*}$ transition c) PCM single point calculations on gas phase optimized geometries.d) PCM calculation on the geometry optimized in aqueous solution e) from ref. 8 f) from ref. 9 


\section{Results of the QM calculations}

S0 optimized geometry at the PBE0/6-31G(d) level in the gas phase:Ura

$\begin{array}{lrrr}6 & -0.023276 & 0.003138 & -0.008074 \\ 6 & 0.001877 & -0.001452 & 1.447218 \\ 6 & 1.182979 & -0.004781 & 2.095848 \\ 7 & 2.376754 & -0.003372 & 1.426544 \\ 6 & 2.483132 & 0.000238 & 0.042557 \\ 7 & 1.257288 & 0.004526 & -0.586544 \\ 8 & 3.550378 & -0.000239 & -0.533504 \\ 8 & -1.015030 & 0.007088 & -0.710354 \\ 1 & -0.941536 & -0.002432 & 1.975716 \\ 1 & 3.256612 & -0.006938 & 1.920553 \\ 1 & 1.287515 & 0.007228 & -1.598645 \\ 1 & 1.257639 & -0.008647 & 3.178302\end{array}$

S0 optimized geometry at the PBE0/6-31G(d) level in the aqueous solution by PCM method: Ura

$\begin{array}{lrcc}6 & -1.194876 & 1.107856 & -0.000259 \\ 6 & 0.019172 & 1.700820 & -0.000052 \\ 7 & 1.176668 & 0.977474 & 0.000007 \\ 6 & 1.210381 & -0.401384 & -0.000112 \\ 7 & -0.040213 & -0.978342 & -0.000464 \\ 6 & -1.282732 & -0.337766 & -0.000041 \\ 8 & 2.248315 & -1.042204 & 0.000203 \\ 8 & -2.314109 & -0.998137 & 0.000352 \\ 1 & -2.110490 & 1.688617 & -0.000213 \\ 1 & 2.081636 & 1.449745 & 0.001373 \\ 1 & -0.054142 & -2.000380 & -0.000343 \\ 1 & 0.149111 & 2.779636 & 0.000214\end{array}$

S0 optimized geometry at the PBE0/6-31G(d) level in the aqueous solution by PCM method:Ura + $4 \mathrm{H} 2 \mathrm{O}$

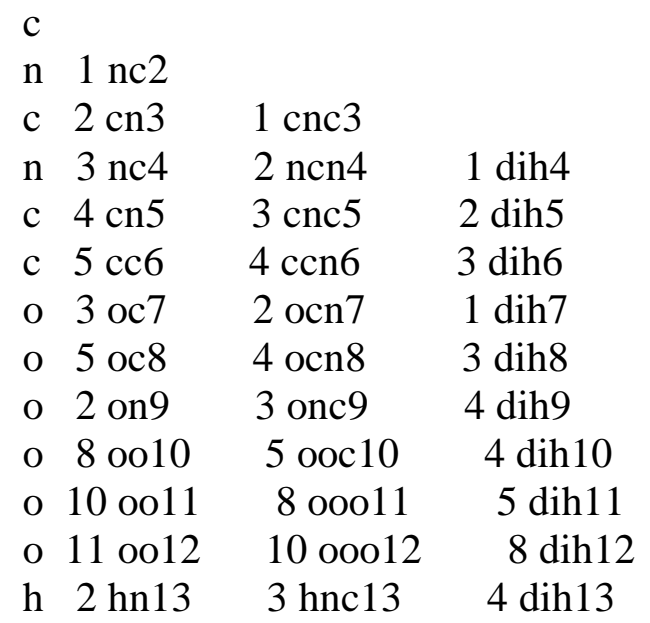




\begin{tabular}{|c|c|c|}
\hline h 4 hn14 & 3 hnc14 & $2 \operatorname{dih} 14$ \\
\hline h 6 hc15 & 5 hcc15 & $4 \operatorname{dih} 15$ \\
\hline h 1 hc16 & 2 hcn16 & $3 \operatorname{dih} 16$ \\
\hline h 9 ho17 & 2 hon17 & $3 \operatorname{dih} 17$ \\
\hline h 9 ho18 & 2 hon18 & $3 \operatorname{dih} 18$ \\
\hline h 12 ho 19 & 11 hoo19 & $10 \operatorname{dih} 19$ \\
\hline h 12 ho 20 & 11 hoo 20 & $10 \operatorname{dih} 20$ \\
\hline h 11 ho 21 & 10 hoo 21 & $8 \operatorname{dih} 21$ \\
\hline h 11 ho 22 & 10 hoo 22 & $8 \operatorname{dih} 22$ \\
\hline h 10 ho23 & 8 hoo23 & $5 \operatorname{dih} 23$ \\
\hline h 10 ho 24 & 8 hoo24 & $5 \operatorname{dih} 24$ \\
\hline $\mathrm{nc} 2$ & 1.364655 & \\
\hline cn3 & 1.376095 & \\
\hline cnc3 & 123.258 & \\
\hline nc4 & 1.375254 & \\
\hline nen4 & 113.797 & \\
\hline $\operatorname{dih} 4$ & -0.004 & \\
\hline cn5 & 1.395404 & \\
\hline cnc5 & 127.494 & \\
\hline $\operatorname{dih} 5$ & -0.030 & \\
\hline $\operatorname{cc} 6$ & 1.445262 & \\
\hline $\operatorname{ccn} 6$ & 114.228 & \\
\hline dih6 & 0.057 & \\
\hline oc7 & 1.224994 & \\
\hline ocn7 & 122.997 & \\
\hline $\operatorname{dih} 7$ & 179.993 & \\
\hline oc8 & 1.230188 & \\
\hline ocn8 & 119.813 & \\
\hline $\operatorname{dih} 8$ & 179.963 & \\
\hline on9 & 2.767959 & \\
\hline onc9 & 95.092 & \\
\hline $\operatorname{dih} 9$ & 179.319 & \\
\hline oo10 & 2.768006 & \\
\hline ooc10 & 129.777 & \\
\hline $\operatorname{dih} 10$ & 15.531 & \\
\hline oo11 & 2.655576 & \\
\hline ooo11 & 82.301 & \\
\hline dih11 & -20.781 & \\
\hline 0012 & 2.807105 & \\
\hline 00012 & 140.041 & \\
\hline $\operatorname{dih} 12$ & 92.266 & \\
\hline hn13 & 1.023422 & \\
\hline hnc13 & 116.455 & \\
\hline $\operatorname{dih} 13$ & 179.930 & \\
\hline hn14 & 1.025008 & \\
\hline hnc14 & 115.759 & \\
\hline dih14 & -179.987 & \\
\hline hc15 & 1.085303 & \\
\hline
\end{tabular}




\begin{tabular}{lc} 
hcc15 & 119.096 \\
dih15 & 179.980 \\
hc16 & 1.087309 \\
hcn16 & 115.201 \\
dih16 & 179.997 \\
ho17 & 0.973975 \\
hon17 & 111.794 \\
dih17 & 99.452 \\
ho18 & 0.975816 \\
hon18 & 74.236 \\
dih18 & 0.547 \\
ho19 & 0.970952 \\
hoo19 & 87.092 \\
dih19 & -107.280 \\
ho20 & 0.974320 \\
hoo20 & 14.202 \\
dih20 & 68.599 \\
ho21 & 0.973727 \\
hoo21 & 107.337 \\
dih21 & -99.839 \\
ho22 & 0.996006 \\
hoo22 & 10.475 \\
dih22 & 179.793 \\
ho23 & 0.982951 \\
hoo23 & 5.412 \\
dih23 & 164.022 \\
ho24 & 0.973804 \\
hoo24 & 106.402 \\
dih24 & -129.122 \\
& \\
\hline
\end{tabular}

$\mathrm{S} 1\left(\mathrm{n} / \pi^{*}\right)$ optimized geometry at the PBE0/6-31G(d) level in the gas phase:Ura

$\begin{array}{lcccccc}\mathrm{C} & & & & & & \\ \mathrm{C} & 1 & 1.399426 & & & & \\ \mathrm{C} & 2 & 1.370246 & 1 & 118.709 & & \\ \mathrm{~N} & 3 & 1.389108 & 2 & 119.987 & 1 & 0.059 \\ \mathrm{C} & 4 & 1.396844 & 3 & 123.765 & 2 & 1.196 \\ \mathrm{~N} & 5 & 1.361227 & 4 & 113.932 & 3 & -1.429 \\ \mathrm{O} & 3 & 1.319260 & 2 & 127.921 & 1 & -179.956 \\ \mathrm{O} & 5 & 1.215224 & 4 & 121.027 & 3 & -181.175 \\ \mathrm{H} & 2 & 1.081210 & 3 & 119.959 & 4 & 179.422 \\ \mathrm{H} & 4 & 1.009190 & 3 & 120.372 & 2 & 177.415 \\ \mathrm{H} & 6 & 1.008640 & 5 & 114.399 & 4 & -178.318 \\ \mathrm{H} & 1 & 1.078380 & 2 & 124.595 & 3 & 179.788\end{array}$


S1(n/ $\left.\pi^{*}\right)$ optimized geometry at the PBE0/6-31G(d) level in the aqueous solution by PCM method: Ura

$\begin{array}{llrr}6 & 0.000000 & 0.000000 & 0.000000 \\ 6 & 0.000000 & 0.000000 & 1.394860 \\ 6 & 1.204251 & 0.000000 & 2.052339 \\ 7 & 2.397053 & -0.006682 & 1.331840 \\ 6 & 2.443654 & -0.012425 & -0.059892 \\ 7 & 1.229301 & -0.009371 & -0.666237 \\ 8 & 1.411380 & -0.000877 & 3.358624 \\ 8 & 3.513014 & -0.018972 & -0.652929 \\ 1 & -0.922783 & -0.004251 & 1.958300 \\ 1 & 3.283643 & 0.032570 & 1.812341 \\ 1 & 1.266557 & -0.040506 & -1.673707 \\ 1 & -0.887700 & -0.003285 & -0.612273\end{array}$

$\mathrm{S} 1\left(\mathrm{n} / \pi^{*}\right)$ optimized geometry at the PBE0/6-31G(d) level in the aqueous solution by PCM method $\mathrm{Ura}+4 \mathrm{H} 2 \mathrm{O}$

$\begin{array}{rrrr}7 & 0.000000 & 0.000000 & 0.000000 \\ 6 & 0.000000 & 0.000000 & 1.357320 \\ 7 & 1.265981 & 0.000000 & 1.937332 \\ 6 & 2.443454 & 0.001549 & 1.192016 \\ 6 & 2.393176 & 0.004354 & -0.179101 \\ 6 & 1.145245 & -0.001191 & -0.802216 \\ 8 & -1.008294 & -0.000622 & 2.049136 \\ 8 & 3.519609 & 0.006688 & 1.960877 \\ 1 & -0.918164 & 0.026965 & -0.416654 \\ 1 & 1.300043 & -0.041331 & 2.945100 \\ 1 & 3.309449 & 0.015002 & -0.752984 \\ 1 & 0.993989 & 0.003654 & -1.869924 \\ 8 & -2.807757 & 0.006980 & -0.154299 \\ 1 & -2.580172 & -0.008948 & 0.795353 \\ 1 & -3.226179 & 0.877081 & -0.284673 \\ 1 & -1.160198 & -0.900170 & 3.816138 \\ 8 & -1.098325 & -1.319386 & 4.690406 \\ 1 & -0.199249 & -1.059312 & 4.959823 \\ 8 & 1.465613 & -0.158737 & 4.787646 \\ 1 & 2.412272 & -0.441817 & 4.922314 \\ 1 & 1.411038 & 0.731660 & 5.178750 \\ 1 & 4.172946 & -0.485905 & 3.783304 \\ 8 & 4.090303 & -0.745807 & 4.728891 \\ 1 & 4.285979 & -1.699698 & 4.729960\end{array}$


S2 $\left(\pi / \pi^{*}\right)$ optimized geometry at the PBE0/6-31G(d) level in the gas phase

Ura

$\begin{array}{lrrr}6 & 0.000000 & 0.000000 & 0.000000 \\ 6 & 0.000000 & 0.000000 & 1.399642 \\ 7 & 1.271145 & 0.000000 & 2.057845 \\ 6 & 2.413056 & -0.049158 & 1.378344 \\ 7 & 2.389430 & -0.098117 & 0.059941 \\ 6 & 1.152203 & -0.027964 & -0.801565 \\ 8 & 3.544078 & -0.057118 & 2.008189 \\ 8 & 1.350596 & 0.008532 & -2.013430 \\ 1 & -0.948935 & 0.016381 & -0.523378 \\ 1 & 1.342176 & 0.056874 & 3.062970 \\ 1 & 3.280955 & -0.150995 & -0.418797 \\ 1 & -0.871052 & 0.013651 & 2.032833\end{array}$

$\mathrm{S} 2\left(\pi / \pi^{*}\right)$ optimized geometry at the PBE0/6-31G(d) level in the aqueous solution by PCM method: Ura

$\begin{array}{rrrr}6 & 0.000000 & 0.000000 & 0.000000 \\ 6 & 0.000000 & 0.000000 & 1.431629 \\ 7 & 1.236772 & 0.000000 & 2.032454 \\ 6 & 2.440708 & -0.050124 & 1.365294 \\ 7 & 2.391714 & -0.095103 & 0.006964 \\ 6 & 1.190528 & -0.041752 & -0.771352 \\ 8 & 3.498937 & -0.055861 & 2.008252 \\ 8 & 1.292864 & -0.033623 & -2.016758 \\ 1 & -0.948935 & 0.016381 & -0.523378 \\ 1 & -0.866011 & 0.030885 & 2.071098 \\ 1 & 1.333473 & 0.056874 & 3.035434 \\ 1 & 3.274309 & -0.148487 & -0.487990\end{array}$

$\mathrm{S} 2\left(\pi / \pi^{*}\right)$ optimized geometry at the PBE0/6-31G(d) level in the aqueous solution by PCM method $\mathrm{Ura}+4 \mathrm{H} 2 \mathrm{O}$

$\begin{array}{lrrr}\mathrm{n} & 2.013044 & -0.595945 & -0.074875 \\ \mathrm{c} & 0.970372 & 0.279778 & 0.132512 \\ \mathrm{n} & -0.282378 & -0.248670 & 0.161662 \\ \mathrm{c} & -0.585606 & -1.643661 & 0.045321 \\ \mathrm{c} & 0.548413 & -2.468217 & -0.174183 \\ \mathrm{c} & 1.880875 & -1.951866 & -0.260732 \\ \mathrm{o} & 1.202734 & 1.487295 & 0.278116 \\ \mathrm{o} & -1.779101 & -1.999763 & 0.146982 \\ \mathrm{~h} & 2.920019 & -0.153447 & -0.062297 \\ \mathrm{~h} & -1.054220 & 0.393629 & 0.297713 \\ \mathrm{~h} & 0.390919 & -3.533484 & -0.296932\end{array}$




$\begin{array}{llrc}\mathrm{h} & 2.778791 & -2.523464 & -0.424635 \\ \mathrm{o} & 4.398707 & 0.799344 & -0.018592 \\ \mathrm{~h} & 4.063249 & 1.703552 & 0.135547 \\ \mathrm{~h} & 4.866706 & 0.582312 & 0.807857 \\ \mathrm{~h} & -0.059357 & 2.920320 & -0.355968 \\ \mathrm{o} & -0.828045 & 3.448968 & -0.627188 \\ \mathrm{~h} & -1.561481 & 2.889810 & -0.314180 \\ \mathrm{o} & -2.459257 & 1.475231 & 0.504989 \\ \mathrm{~h} & -3.189552 & 0.869454 & 0.198181 \\ \mathrm{~h} & -2.628038 & 1.617049 & 1.453754 \\ \mathrm{~h} & -3.255819 & -1.102215 & -0.073791 \\ \mathrm{o} & -4.040963 & -0.521244 & -0.194348 \\ \mathrm{~h} & -4.222804 & -0.566065 & -1.149928\end{array}$

